\title{
Upper bounds on sparticle masses from muon $g-2$ and the Higgs mass and the complementarity of future colliders
}

\author{
Marcin Badziak, Zygmunt Lalak, Marek Lewicki, Marek Olechowski \\ and Stefan Pokorski \\ Institute of Theoretical Physics, Faculty of Physics, University of Warsaw, \\ ul. Pasteura 5, PL-02-093 Warsaw, Poland \\ E-mail: mbadziak@fuw.edu.pl, Zygmunt.Lalak@fuw.edu.pl, \\ Marek.Lewicki@fuw.edu.pl, Marek.Olechowski@fuw.edu.pl, \\ Stefan.Pokorski@fuw.edu.pl
}

ABSTRACT: Supersymmetric (SUSY) explanation of the discrepancy between the measurement of $(g-2)_{\mu}$ and its SM prediction puts strong upper bounds on the chargino and smuon masses. At the same time, lower experimental limits on the chargino and smuon masses, combined with the Higgs mass measurement, lead to an upper bound on the stop masses. The current LHC limits on the chargino and smuon masses (for not too compressed spectrum) set the upper bound on the stop masses of about $10 \mathrm{TeV}$. The discovery potential of the future lepton and hadron colliders should lead to the discovery of SUSY if it is responsible for the explanation of the $(g-2)_{\mu}$ anomaly. This conclusion follows from the fact that the upper bound on the stop masses decreases with the increase of the lower experimental limit on the chargino and smuon masses.

Keywords: Supersymmetry Phenomenology

ARXIV EPRINT: 1411.1450 


\section{Contents}

1 Introduction 1

2 SUSY contribution to $(g-2)_{\mu} \quad 3$

3 Upper bounds on the chargino and smuon masses from $(g-2)_{\mu} \quad 4$

4 Upper bounds on the stop masses $\quad 6$

5 Conclusions 10

$\begin{array}{ll}\text { A Bino contribution } & 11\end{array}$

\section{Introduction}

For many years supersymmetric (SUSY) particles were expected to be light and accessible to the near-future experiments. The main argument behind those expectations was the supersymmetric solution to the hierarchy problem of the Standard Model. However, negative results of searches for SUSY in the first run of the LHC pushed naturalness arguments to the edge. While Natural Supersymmetry [1-3] is not yet excluded by the LHC some degree of fine-tuning must be present in supersymmetric models [4-6]. Therefore, it is time to ask if there are good reasons to expect relatively light SUSY particles without invoking the naturalness arguments. This question was already raised more than ten years ago when split SUSY has been invented. For a good supersymmetric dark matter candidate and for the gauge coupling unification gauginos should be relatively light $[7,8]$. More recently, there have been attempts to set an upper bound on supersymmetric mass scale using some theoretical [9] or phenomenological [10] arguments without relying on naturalness. In this paper, we focus on the anomalous magnetic moment of muon and calculate upper bounds on the superpartner masses under the assumption that supersymmetry explains the apparent excess in the measured value [11] of this observable over the Standard Model (SM) prediction [12-14].

It is well known that the supersymmetric contribution to $(g-2)_{\mu}$ depends mainly on the electroweak (EW) part of the SUSY spectrum and that it grows with $\tan \beta$ [15]. A detailed analysis of that contribution was performed in ref. [16] but the upper limits on the chargino and smuon masses still consistent with the present experimental value of $(g-2)_{\mu}$ have not been explicitly presented there. Moreover, both the experimental and the SM results have changed since the publication of ref. [16]. One of the aims of the present paper is to find those up-to-date upper bounds as a function of $\tan \beta$. We find these upper bounds in the framework of the MSSM in a model-independent way i.e. without assuming any particular mechanism of supersymmetry breaking. 
We also point out that the value of $(g-2)_{\mu}$ gives constraints not only on the slepton sector but indirectly also on the squark sector. The upper bounds on the smuon and chargino masses depend on $\tan \beta$ so the present (or future) experimental lower limits on these sparticle masses can be translated into a lower bound on $\tan \beta$. Since the tree-level Higgs mass grows with $\tan \beta$, a lower bound on $\tan \beta$ results in an upper bound on the size of the loop corrections to the Higgs mass, from not overshooting the measured value of about $125 \mathrm{GeV}$. This in turn leads to an upper bound on the stop masses since they dominate the radiative corrections to the Higgs mass. ${ }^{1}$

We find that the present LEP limits on the smuon and chargino masses together with the requirement of e.g. $1 \sigma$ agreement with $(g-2)_{\mu}$ imply $\tan \beta \gtrsim 2 .^{2}$ This gives rather weak upper bound on the stop masses of about $10^{3}-10^{4} \mathrm{TeV}$. However, even a slight improvement in the experimental limits on the smuon and chargino masses would lead to a substantial improvement of the lower bound on $\tan \beta$ and, in turn, to a strong upper bound on the stop masses of order $\mathcal{O}(10 \mathrm{TeV})$. We emphasize the complementarity of the future hadron and lepton colliders in testing the SUSY solution to the $(g-2)_{\mu}$ anomaly.

Implications of the Higgs mass measurement for the solution to the $(g-2)_{\mu}$ anomaly have been studied in various classes of SUSY models. It has been realized that it is difficult to reconcile the $(g-2)_{\mu}$ measurement with the $125 \mathrm{GeV}$ Higgs in the simplest models of SUSY breaking, see e.g. ref. [20]. However, it has been noted that $(g-2)_{\mu}$ can be in agreement with the experimental result in GUT models with non-universal gaugino masses [2124]. The motivation of those papers was to reconcile the $(g-2)_{\mu}$ measurement with the higgs mass measurement in concrete models, so it was different from our motivation. In each model, the authors obtain certain range of acceptable squark and gluino masses, as a consequence not only of the Higgs mass measurement but also due to correlations between various soft terms imposed by the model and the renormalization group running between the GUT and EW scale. Since in the present paper we do not impose any such correlations between the soft terms the upper bounds on the stop masses that we have found are more conservative (i.e. weaker) than those found in the GUT models with non-universal gaugino masses. ${ }^{3}$ Moreover, in contrast to refs. [21-24] we do not impose any constraints for thermal relic density of the LSP allowing this way for non-standard cosmological history. Interestingly, we found that even under these conservative assumptions the SUSY solution to the $(g-2)_{\mu}$ can be tested in the realistic future colliders.

The paper is organized as follows. In section 2 we briefly review the dominant SUSY contributions to $(g-2)_{\mu}$. In section 3 we calculate the upper bounds on the smuon and chargino masses as a function of $\tan \beta$. In section 4 we calculate the upper bound on the stop masses from the measured Higgs mass as a function of $\tan \beta$ and combine these

\footnotetext{
${ }^{1}$ The upper bounds on the stop masses as a function of $\tan \beta$ have been discussed in refs. [17, 18] in the context of split supersymmetry.

${ }^{2}$ The bound may not be valid if the higgsino mass is at least order of magnitude larger than the slepton and gaugino masses (see the appendix and ref. [19]).

${ }^{3}$ Non-universal gaugino masses in GUT models can originate from GUT-nonsinglet SUSY breaking $F$ terms [25]. Such $F$-terms typically result also in non-universal soft scalar masses and trilinear terms [26]. This was not taken into account in refs. [21-24] so the upper bounds on the coloured sparticles in general GUT model may be weaker than those found in refs. [21-24].
} 
results with those of the preceding section to obtain an upper bound on the stop masses as a function of lower experimental limits on smuon and chargino masses. Finally, we summarize our results in section 5 .

\section{SUSY contribution to $(g-2)_{\mu}$}

The discrepancy between the experimental value from BNL [11] and the SM prediction is above $3 \sigma$. The theoretical prediction in the SM has been evaluated by several different groups [12-14] and the obtained results are in a very good agreement between those groups. For the sake of definiteness, in this paper we use the result from ref. [12] which leads to the following discrepancy between the SM prediction and the experiment:

$$
\Delta a_{\mu} \equiv a_{\mu}^{\exp }-a_{\mu}^{\text {th }}=(28.7 \pm 8.0) \times 10^{-10},
$$

where the uncertainty is the combination of the experimental and theoretical ones. This discrepancy is similar to the SM electroweak contribution. SUSY can account for this discrepancy because the contribution from the SUSY EW sector is enhanced by $\tan \beta$. This fact is crucial for our discussion of the upper bound on the stop masses.

The leading supersymmetric contribution to the muon anomalous magnetic moment can be approximated by $[16,27]$ the chargino-sneutrino contribution

$$
a_{\mu}^{\chi^{ \pm}}=\frac{\alpha m_{\mu}^{2} \mu M_{2} \tan \beta}{4 \pi \sin ^{2} \theta_{W} m_{\tilde{\nu}_{\mu}}^{2}}\left(\frac{f_{\chi^{ \pm}}\left(M_{2}^{2} / m_{\tilde{\nu}_{\mu}}^{2}\right)-f_{\chi^{ \pm}}\left(\mu^{2} / m_{\tilde{\nu}_{\mu}}^{2}\right)}{M_{2}^{2}-\mu^{2}}\right)
$$

and the bino-smuon contribution

$$
a_{\mu}^{\chi^{0}}=\frac{\alpha m_{\mu}^{2} M_{1}\left(\mu \tan \beta-A_{\mu}\right)}{4 \pi \cos ^{2} \theta_{W}\left(m_{\tilde{\mu}_{R}}^{2}-m_{\tilde{\mu}_{L}}^{2}\right)}\left(\frac{f_{\chi^{0}}\left(M_{1}^{2} / m_{\tilde{\mu}_{R}}^{2}\right)}{m_{\tilde{\mu}_{R}}^{2}}-\frac{f_{\chi^{0}}\left(M_{1}^{2} / m_{\tilde{\mu}_{L}}^{2}\right)}{m_{\tilde{\mu}_{L}}^{2}}\right),
$$

where $m_{\tilde{\mu}_{L}}$ and $m_{\tilde{\mu}_{R}}$ are smuon soft masses, and the loop functions are given by

$$
\begin{aligned}
f_{\chi^{ \pm}}(x) & =\frac{x^{2}-4 x+3+2 \ln x}{(1-x)^{3}}, & & f_{\chi^{ \pm}}(1)=-2 / 3, \\
f_{\chi^{0}}(x) & =\frac{x^{2}-1-2 x \ln x}{(1-x)^{3}}, & f_{\chi^{0}}(1) & =-1 / 3 .
\end{aligned}
$$

In the majority of the MSSM parameter space the chargino-sneutrino contribution (2.2) dominates over all other SUSY contributions. This contribution decouples when muon sneutrino or chargino masses get large. Nevertheless, even if these masses are many times larger than the $\mathrm{W}$ boson mass this contribution can be of the order of the SM EW contribution because the mass suppression can be compensated by large values of $\tan \beta$. Therefore, the upper bounds on slepton and chargino masses obtained from the requirement of fitting the value of $(g-2)_{\mu}$ grow with $\tan \beta$. The next section is devoted to the calculation of these upper bounds. 


\section{Upper bounds on the chargino and smuon masses from $(g-2)_{\mu}$}

In this section we calculate the upper bounds on the chargino and slepton masses as a function of $\tan \beta$. To this end we perform a scan over the relevant MSSM parameters. As discussed in the previous section, the SUSY contribution to $(g-2)_{\mu}$ depends dominantly on $\tan \beta$, soft gaugino masses $M_{1}, M_{2}$, the smuon and sneutrino soft mass terms (for the first slepton family we take them equal to the second one), $m_{E_{1}}=m_{E_{2}}$ and $m_{L_{1}}=m_{L_{2}}$, and the $\mu$ parameter, so we vary them in the following ranges:

$$
\begin{aligned}
1.5 & \leq \tan \beta \\
0 \mathrm{GeV} & \leq \quad\left|M_{1}\right| \leq 150, \\
40 \mathrm{GeV} & \leq\left|M_{2}\right| \leq 1500 \mathrm{GeV} \\
90 \mathrm{GeV} & \leq m_{L_{2}}, m_{R_{2}} \leq 1500 \mathrm{GeV} \\
50 \mathrm{GeV} & \leq \quad|\mu| \leq 1500 \mathrm{GeV} .
\end{aligned}
$$

The dependence on other supersymmetric parameters is weak, therefore we fix all the squark masses and the gluino mass at $2.5 \mathrm{TeV}$, which satisfy the current LHC limits. We also set all the trilinear terms to zero. Finally, we set $m_{A}=1 \mathrm{TeV}$ and the stau soft masses $m_{L_{3}}=m_{E_{3}}=500 \mathrm{GeV}$. We calculate the full one loop and the leading two-loop supersymmetric contributions to the muon anomalous magnetic moment, given in ref. [16]. In the two loop contribution we set $M_{\text {SUSY }}$ (defined in ref. [16]) to the bino or smuon mass, whichever is lighter.

The largest positive SUSY contribution to $(g-2)_{\mu}$ is obtained when $\mu, M_{1}$ and $M_{2}$ have the same sign because then both the chargino-sneutrino (2.2) and bino-smuon (2.3) contributions are positive. We have confirmed it by scanning over all possible sign assignments of $\mu, M_{1}$ and $M_{2}$.

In the left panel of figure 1 the upper bounds on the masses of the lighter chargino and smuon consistent with the $(g-2)_{\mu}$ measurement at $1 \sigma$ level are presented. The bounds result from the requirement that the full $a_{\mu}$ (with SM and SUSY contributions taken into account) differs from the experimental central value by at most one standard deviation given in eq. (2.1). The results depend on values of $\tan \beta$. For large $\tan \beta \sim \mathcal{O}(50)$, the lightest smuon masses up to $1 \mathrm{TeV}$ may be sufficient to explain the $(g-2)_{\mu}$ anomaly. It can be also seen that with the LEP bounds for the chargino and smuon mass of 103.5 and $100 \mathrm{GeV}$ [28-33], respectively, the $(g-2)_{\mu}$ anomaly can be explained in the MSSM with $\tan \beta \gtrsim 2$.

The performed scan gives bounds not only on the chargino and smuon masses but also on masses of bino and muon sneutrino. However, we concentrate on the charged SUSY particles because the existing experimental limits on their masses are much stronger than in the case of neutral particles.

The LHC limits on the chargino and smuon masses are not as generic as the LEP ones but in certain scenarios they are much stronger. In the easiest (from the experimental point of view) case with massless LSP and the slepton masses two times smaller than the chargino mass, the latter is excluded up to about $700 \mathrm{GeV}$ [34-36]. For such a spectrum 

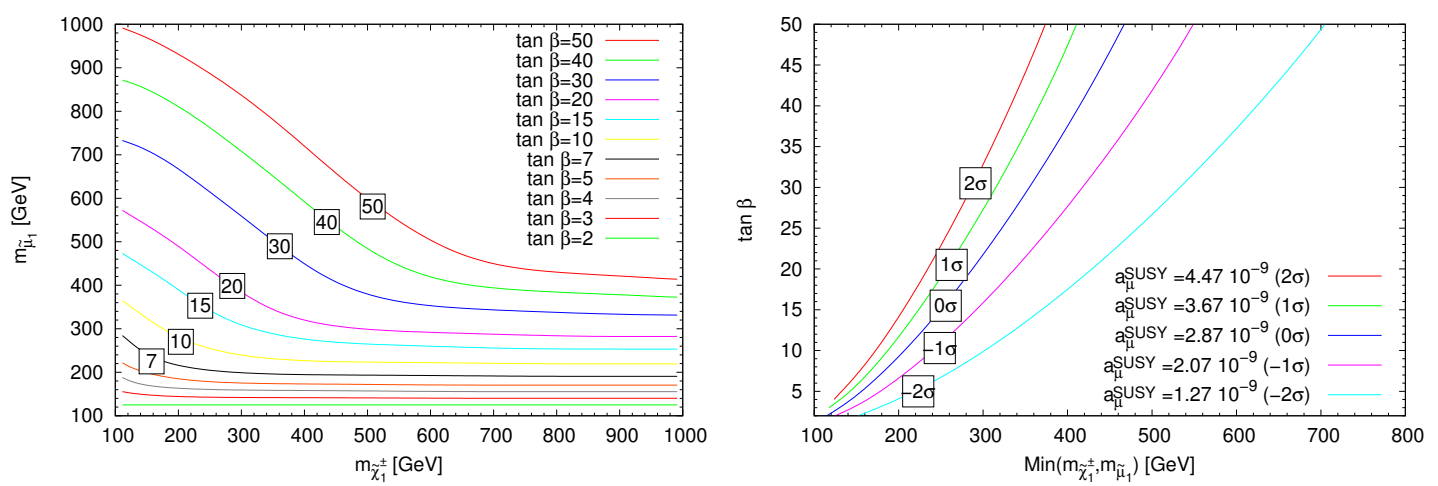

Figure 1. Left: upper bounds on the lightest chargino and smuon masses for several values of $\tan \beta$ obtained from the requirement of accommodating the experimental result for $(g-2)_{\mu}$ with $1 \sigma$ accuracy. Right: lower bounds on $\tan \beta$ as a function of a common (hypothetical) experimental lower bound on the chargino and smuon masses obtained from the requirement of getting $a_{\mu}^{\mathrm{SUSY}}=$ $\Delta a_{\mu}, \Delta a_{\mu} \pm 1 \sigma, \Delta a_{\mu} \pm 2 \sigma$ (see eq. (2.1)).

large values of $\tan \beta \gtrsim 30$ are required to explain the $(g-2)_{\mu}$ anomaly. However, currently the LHC sets no constraints for the spectra with mass-degeneracies smaller than about $10 \%$. The situation might be improved with monojet searches and it was argued that with $300 \mathrm{fb}^{-1}$ at the $14 \mathrm{TeV}$ LHC charginos can be excluded up to $200 \mathrm{GeV}$ even for compressed spectra [37].

In the left panel of figure 1 the predicted $(g-2)_{\mu}$ agrees at $1 \sigma$ with the experimental result. However, for a more complete qualitative picture of the bounds that would account for the theoretical uncertainties in the SM calculations and potential improvements in the experimental precision, ${ }^{4}$ in the right panel of figure 1 we also plot a lower bound on $\tan \beta$ as a function of a common (hypothetical) experimental lower bound on the chargino and smuon masses. Those bounds are obtained from the requirement of getting $a_{\mu}^{\mathrm{SUSY}}=$ $\Delta a_{\mu}, \Delta a_{\mu} \pm 1 \sigma, \Delta a_{\mu} \pm 2 \sigma$ (see eq. (2.1)).

Let us also comment that at the two-loop level the SUSY contribution to $(g-2)_{\mu}$ depends also on parameters other than those varied in our numerical scan. It was recently shown that for large sfermion masses the dominant two-loop contribution comes from the fermion/sfermion loop [40]. That contribution does not decouple with the sfermion masses and is logarithmically enhanced. It was shown in ref. [40] that this two-loop contribution is about $5(10) \%$ of the one-loop contribution for the squark masses of order 10 (1000) TeV. Since the latter is approximately linear in $\tan \beta$, the inclusion of this correction would shift the lower bounds on $\tan \beta$ also by about $5(10) \%$ for the squark masses of order 10 (1000) $\mathrm{TeV}$. Therefore, the impact of the two-loop corrections on the upper bound for the slepton and chargino masses is relatively small.

The results presented so far in this section assumed the higgsino masses below $1.5 \mathrm{TeV}$. However, the results are not affected very much if $\mu$ is scanned up to values larger by a factor of a few. This can be seen from figure 2 where the upper bounds on the masses of

\footnotetext{
${ }^{4}$ Two new $g-2$ experiments at Fermilab [38] and JPARC [39] are expected to start collecting data around 2017.
} 


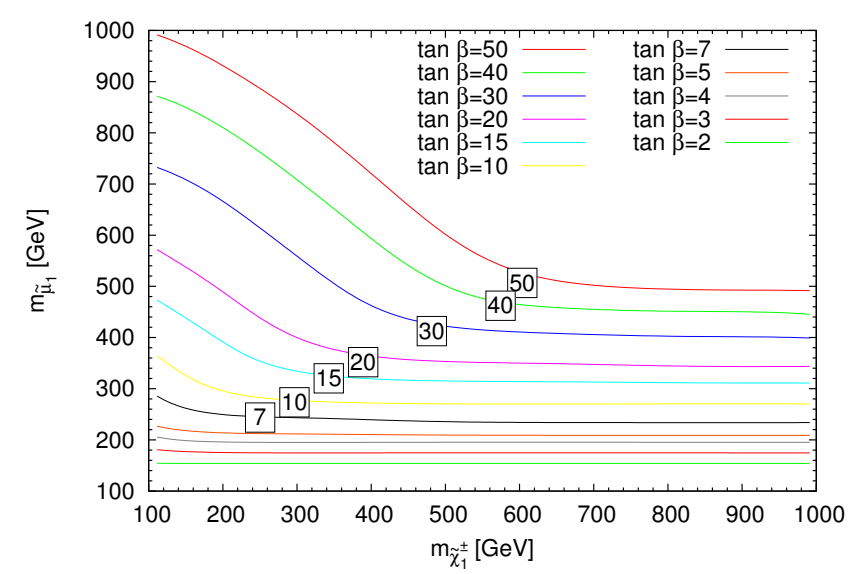

Figure 2. Same as in the left panel of figure 1 but for the upper bound on $|\mu|$ increased to $3 \mathrm{TeV}$ (ranges of other parameters as in (3.1)).

the lighter chargino and smuon are presented assuming $|\mu| \leq 3 \mathrm{TeV}$. For smaller masses of $\chi_{1}^{ \pm}$the effect of larger $\mu$ on the upper bound on the smuon mass is hardly visible because a heavy higgsino suppresses the usually dominant chargino-sneutrino contribution. In the large $\chi_{1}^{ \pm}$region of the plot the upper bound on the smuon mass is weakened by about $20-25 \%$. This is because the bino contribution $(2.3)$ to $(g-2)_{\mu}$ is inversely proportional to the third power of smuon masses (after taking into account that $M_{1} \sim m_{\tilde{\mu}_{1}}$ required to avoid the suppression of the bino contribution by the loop function $f_{\chi^{0}}(x)$ ) but is only linear in the $\mu$ parameter.

\section{Upper bounds on the stop masses}

It is clear from the previous section that the lower limit on the smuon and chargino masses translates into a lower bound on $\tan \beta$, if the $(g-2)_{\mu}$ anomaly is to be explained by supersymmetric contributions. It is well known that such a bound can be translated into an upper bound on the stop masses [17, 18].

Firstly, we recalculate the upper bound on the stop masses as a function of $\tan \beta$. The one-loop formula for the Higgs mass in the MSSM reads:

$$
m_{h}^{2} \approx M_{Z}^{2} \cos ^{2} 2 \beta+\frac{3 g^{2} m_{t}^{4}}{8 \pi^{2} m_{W}^{2}}\left[\ln \left(\frac{M_{\tilde{t}}^{2}}{m_{t}^{2}}\right)+\frac{X_{t}^{2}}{M_{\tilde{t}}^{2}}\left(1-\frac{X_{t}^{2}}{12 M_{\tilde{t}}^{2}}\right)\right],
$$

where $M_{\tilde{t}} \equiv \sqrt{m_{\tilde{t}_{1}} m_{\tilde{t}_{2}}}\left(m_{\tilde{t}_{i}}\right.$ are the eigenvalues of the stop mass matrix) and $X_{t} \equiv A_{t}-$ $\mu / \tan \beta$ with $A_{t}$ being the SUSY breaking top trilinear coupling. For given stop masses the Higgs mass is minimal for vanishing stop-mixing parameter $X_{t}$ so, being interested in the upper bound on the stop masses, we set $X_{t}=0 .{ }^{5}$

\footnotetext{
${ }^{5}$ For very large values of $X_{t}>\sqrt{12} M_{\tilde{t}}$ the correction from the stop mixing becomes negative with its absolute value increasing very rapidly with the ratio $X_{t} / M_{\tilde{t}}$. Therefore, in principle one can get $m_{h}=$ $125 \mathrm{GeV}$ even if the logarithmic contribution overshoots the measured Higgs mass. That would require a big fine-tuning of $X_{t} / M_{\tilde{t}}$ and, more importantly, lead to the EW vacuum destabilization [41-43].
} 
For the calculation of the Higgs mass we use FeynHiggs 2.10.0 [44, 45] which combines the existing fixed-order results for the radiative corrections up to two loops with a resummation of the leading and subleading logarithmic contributions from stops to all orders. The inclusion of the latter allows for a reliable prediction of the Higgs mass also for stops much heavier than the TeV scale.

The experimental precision of the Higgs mass measurement at the LHC has reached several hundreds MeV. The latest results from ATLAS [46] and CMS [47] read:

$$
\begin{aligned}
m_{h}^{\text {ATLAS }} & =125.36 \pm 0.37 \text { (stat.) } \pm 0.18 \text { (syst.) } \mathrm{GeV}, \\
m_{h}^{\mathrm{CMS}} & =125.03_{-0.27}^{+0.26} \text { (stat.) }{ }_{-0.15}^{+0.13} \text { (syst.) GeV } .
\end{aligned}
$$

A simple combination of the above results (assuming Gaussian errors) gives:

$$
m_{h}^{\exp }=125.14 \pm 0.24 \mathrm{GeV} .
$$

With this experimental precision the dependence of the Higgs mass on other than stops sparticle masses (mainly gauginos and higgsinos) has to be taken into account. The explanation of the $(g-2)_{\mu}$ anomaly calls for rather light charginos. On the other hand, we would like to find a conservative upper bound on the stop masses so in the following analysis we fix $M_{2}=\mu=1 \mathrm{TeV}$. Lighter charginos would result in a larger Higgs mass, hence, a more stringent upper bound on the stop masses. For example, we find that for $M_{2}=\mu=200 \mathrm{GeV}$ the Higgs mass is typically bigger by about $1.5 \mathrm{GeV}$ than in the case $M_{2}=\mu=1 \mathrm{TeV}$. We also fix $M_{3}=2.5 \mathrm{TeV}$ in order to be on the safe side from the LHC gluino mass bounds. We have checked that increasing $M_{3}$ up to $M_{\tilde{t}}$ decreases the prediction for the Higgs mass only by several hundreds of MeV.

The last parameter whose value has a non-negligible impact on the Higgs mass is the pseudoscalar Higgs mass, $m_{A}$, because it controls the mixing between the SM-like and the heavy MSSM Higgs. Smaller values of $m_{A}$ result in a smaller Higgs mass so, in order to be conservative, we use the values of $m_{A}$ equal to the current experimental lower limits. For $\tan \beta \gtrsim 15$, the best limit comes from the Higgs searches in the $\tau \tau$ channel performed by ATLAS [48] and CMS [49]. It varies from about $400 \mathrm{GeV}$ for $\tan \beta=15$ up to $950 \mathrm{GeV}$ for $\tan \beta=50$. For a smaller $\tan \beta$, the main constraint comes from the LHC Higgs coupling measurements which set the limit $m_{A} \gtrsim 400 \mathrm{GeV}$ almost independently of $\tan \beta$ for $\tan \beta \gtrsim 2[50]$ required to explain the $(g-2)_{\mu}$ anomaly. We have found that for $m_{A}=400 \mathrm{GeV}$ the Higgs mass is smaller by about $2 \mathrm{GeV}$ than in the case of decoupled $A$.

In figure 3 we plot the Higgs mass versus $M_{\tilde{t}}$ for several values of $\tan \beta$ with the remaining MSSM parameters set to the values described above. In the calculation we use the top mass from the recent combination of the LHC and Tevatron results which yields $m_{t}=173.34 \pm 0.76 \mathrm{GeV}$ [51]. The upper bound on $M_{\tilde{t}}$ is below $25 \mathrm{TeV}$ even for $\tan \beta=5$. For $\tan \beta=10$, the upper bound is about $6 \mathrm{TeV}$ using the central values of the FeynHiggs prediction and the measured values of the Higgs and top masses. After taking into account the theoretical uncertainty reported by FeynHiggs (about $1 \mathrm{GeV}$ for $M_{\tilde{t}} \approx 10 \mathrm{TeV}$ ), ${ }^{6}$ using the top mass $1 \sigma$ below the central value (which reduces the Higgs mass by about $0.7 \mathrm{GeV}$ )

\footnotetext{
${ }^{6}$ An improved calculation of the Higgs mass was performed recently also in refs. [52, 53]. It was noted
} 

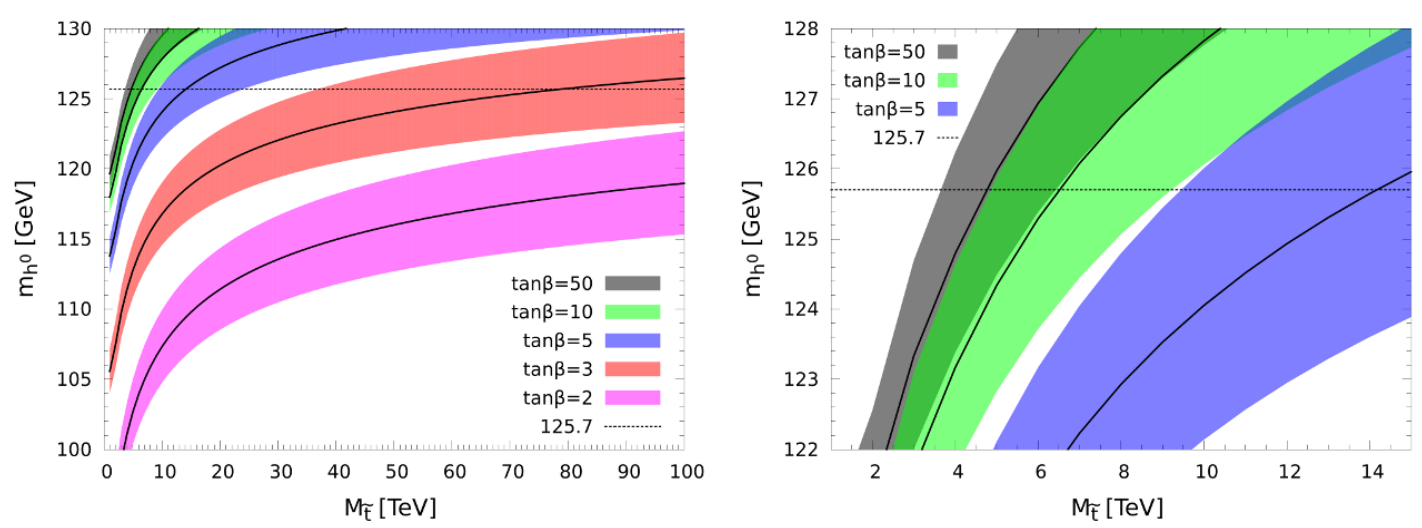

Figure 3. Left: the Higgs mass versus $M_{\tilde{t}}$ for several values of $\tan \beta$. Other relevant MSSM parameters are: $X_{t}=0, M_{3}=2.5 \mathrm{TeV}, M_{2}=\mu=1 \mathrm{TeV}$ and $m_{A}$ is set to the current lower experimental limit, see the text for more details. The width of the bands corresponds to the theoretical uncertainty, as calculated by FeynHiggs, added linearly to the uncertainty from varying the top mass within $1 \sigma$ from the experimental central value. Right: zoom of the plot on the left.

and imposing the Higgs mass of 125.7 (which is $2 \sigma$ above the central value) the upper bound on the stop masses for $\tan \beta=10$ is relaxed to about $9 \mathrm{TeV}$.

We can combine now the results shown in figure 1 with the Higgs mass dependence on $\tan \beta$ and the stop masses, for the vanishing stop mixing. In the left panel of figure 4 we plot the contours of the upper bounds on the stop masses in the plane of the hypothetical experimental lower bounds on the lightest chargino and smuon masses, if one requires consistency with the $(g-2)_{\mu}$ measurement at $1 \sigma$ level. In this plot we take the experimental upper bound on the Higgs mass at $95 \%$ C.L. which is, according to eq. (4.4), about $125.7 \mathrm{GeV}$. In the theoretical prediction for the Higgs mass we use $\mu=M_{2}=1 \mathrm{TeV}$, $m_{A}$ equal to current experimental lower limit and take into account the theoretical uncertainties reported by FeynHiggs (in order to get conservative upper bound we assume that FeynHiggs overestimate the Higgs mass). Moreover, we use the value of the top mass, $m_{t}=172.58 \mathrm{GeV}$, which is $1 \sigma$ below the current experimental central value. With these conservative numbers we find that the LEP constraints set the upper bound on the stop masses of about $7000 \mathrm{TeV} .^{7}$

The left panel of figure 4 demonstrates that relatively mild improvements of the limits on the chargino and smuon masses would have a strong impact on the upper bound on the stop masses. The reason is that the tree-level contribution to the Higgs mass strongly depends on $\tan \beta$ as long as $\tan \beta$ is not large. While the LHC limits are not generic, for typical spectra the smuon and chargino masses are excluded at least up to $300 \mathrm{GeV}$ [54].

in ref. [53] that their prediction of the Higgs mass (with the theoretical uncertainty estimated to be about $1 \mathrm{GeV}$ ) for the stop masses of $10 \mathrm{TeV}$ is about $3 \mathrm{GeV}$ smaller than the corresponding prediction of FeynHiggs for the same SUSY spectrum.

${ }^{7}$ The exact value of this upper bound is quite sensitive to the assumption about the values of $\mu, M_{2}$, $m_{A}, m_{t}$ but it is typically in the range between $10^{3}$ and $10^{4} \mathrm{TeV}$. This partly stems from the fact that for such heavy stops the theoretical uncertainty of FeynHiggs exceeds $3 \mathrm{GeV}$ and grows with $M_{\tilde{t}}$ only slightly slower than the central value returned by FeynHiggs. 

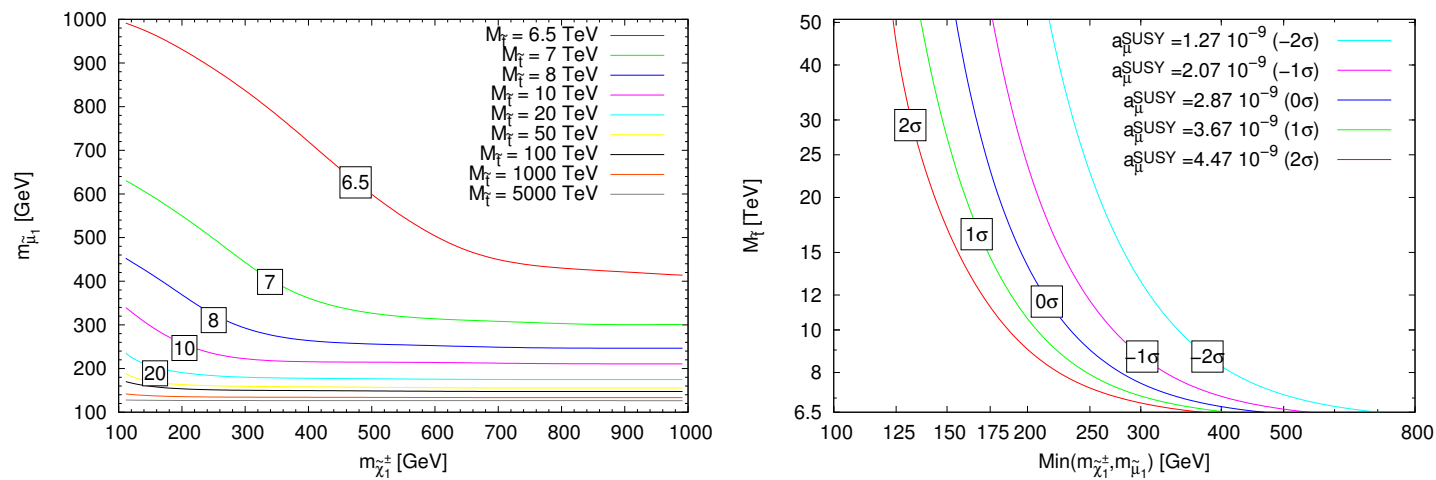

Figure 4. Left: the contours of the upper bounds on the stop masses in the plane of hypothetical experimental lower bounds on the lightest chargino and smuon masses and requiring the prediction for $(g-2)_{\mu}$ to be within $1 \sigma$ from its experimental value. Right: the upper bound on the stop masses as a function of a common (hypothetical) experimental lower bound on the chargino and smuon masses for several values of $a_{\mu}^{\text {SUSY }}$. The values of $a_{\mu}^{\text {SUSY }}$ are as in figure 1.

This is enough to bring down the upper bound on the stop masses to about $8 \mathrm{TeV}$.

An electron-positron collider with $\sqrt{s}=500 \mathrm{GeV}$, which is a designed center-of-mass energy at ILC [55] and the upgraded TLEP [56] would probe chargino and slepton masses up to about $250 \mathrm{GeV}$ bringing down the robust upper bound on the stop masses to around $10 \mathrm{TeV}$. Since the tree-level Higgs mass is saturated for large $\tan \beta$ it is difficult to reduce the upper bound on the stop masses far below $10 \mathrm{TeV}$, as it is evident from the left panel of figure 4 .

In addition to the improvement in the stop mass bounds from better lower limits for the smuon and chargino masses, a slightly better precision may come from stronger limits on $m_{A}$ and from improvements in the Higgs and top mass measurements.

Similarly as in figure 1, for a broader qualitative picture of the upper bounds on the stop masses, it is also interesting to see how they change if different experimental values of $(g-2)_{\mu}$ are taken. Thus, in the right panel of figure 4 we plot the upper bound on the stop masses as a function of a common hypothetical experimental lower limits on the smuon and chargino masses for several values of $a_{\mu}^{\mathrm{SUSY}}$. This plot is especially interesting since future lepton colliders are expected to set similar experimental lower limits on both masses, roughly equal to a half of the center of mass energy of the colliding leptons. Assuming that the theoretical $(g-2)_{\mu}$ is consistent with the current measurement at $2 \sigma$, the upper bound on the stop masses is somewhat relaxed. However, if the lower experimental limit on the chargino and smuon masses was set at around $300 \mathrm{GeV}$ even the $2 \sigma$ agreement with the current $(g-2)_{\mu}$ measurement would imply the upper bound on the stop masses around $10 \mathrm{TeV}$.

The stops with masses around $10 \mathrm{TeV}$ are beyond the LHC reach. While precise studies of the discovery potential of the $100 \mathrm{TeV}$ hadron collider are still missing, preliminary simulations indicate that such masses could be probed at that collider provided that gluinos and other squarks are in a similar mass range [57]. ${ }^{8}$ A direct production of $10 \mathrm{TeV}$ stops

\footnotetext{
${ }^{8}$ It was recently argued, using Bayesian statistics to fit all the available data including $(g-2)_{\mu}$, that
} 
is, of course, more challenging. Nevertheless, in the recent article [59] it is argued that directly produced stops decaying to a top and a neutralino could be discovered (excluded) up to 6.5 (8) $\mathrm{TeV}$ with $3000 \mathrm{fb}^{-1}$ of integrated luminosity at the $100 \mathrm{TeV}$ collider.

In the NMSSM [60] the upper bounds on the smuon, chargino and stop masses from $(g-2)_{\mu}$ and the Higgs mass are typically similar to the MSSM ones. The value of $(g-2)_{\mu}$ in the NMSSM can be enhanced, as compared to MSSM, only for a very light NMSSM-like $\mathrm{CP}$-odd Higgs with the mass in the range of $5-20 \mathrm{GeV}$ [61]. However, this contribution is non-negligible only for a large $\tan \beta$ (in order to explain $(g-2)_{\mu}$ at $1 \sigma(2 \sigma)$ with that contribution alone the $\tan \beta$ at least about 50 (30) is needed) so the left panel of figure 1 remains valid in the NMSSM for most values of $\tan \beta$. The upper bounds on the stop masses discussed above hold for the NMSSM if the mixing between the SM-like Higgs and the NMSSM singlet-like scalar is neglected. However, these upper bounds can disappear if the singlet-like scalar is heavier than the SM-like Higgs (but not decoupled) because then their mixing gives negative contribution to the Higgs mass and may cancel a too large logarithmic correction from very heavy stops.

Another point worth emphasizing is that SUSY spectrum consistent with the $(g-2)_{\mu}$ measurement does not have to be much split, especially for large values of $\tan \beta$. For large $\tan \beta$, all slepton and chargino masses can be above $500 \mathrm{GeV}$, while stops can be around $1 \mathrm{TeV}$ if a large stop mixing is present [62] and/or a mixing with additional light singlet-like scalar is introduced [63], as in the NMSSM.

\section{Conclusions}

We have investigated upper bounds on the sparticle masses originating from the synergy between the $(g-2)_{\mu}$ and the Higgs mass measurements. If SUSY is responsible for the $(g-2)_{\mu}$ anomaly, the chargino and smuon masses are strongly constrained from above, with the bound being stronger for smaller values of $\tan \beta$. In consequence, experimental lower limits on the chargino and smuon masses lead to lower bounds on $\tan \beta$. We have translated the bounds on $\tan \beta$ into upper bounds on the stop masses from the requirement that the predicted Higgs mass does not overshoot the experimental value. The main results of this paper are presented in figure 4. The LEP limits on the smuon and chargino masses result in an upper bound on the stop masses exceeding $10^{3} \mathrm{TeV}$. However, even mild improvement of the LEP limits results in a significant improvement of this upper bound. Current LHC limits on smuon and chargino masses obtained for not too compressed gaugino and higgsino spectra reduce the upper bound on the stop masses to about $10 \mathrm{TeV}$. Electron-positron colliders operating at $\sqrt{s}=500(1000) \mathrm{GeV}$ would allow to set the upper bound on the stop masses to about 10 (5) TeV. Such stops could be discovered at the $100 \mathrm{TeV}$ hadron collider.

The main conclusion of this paper is that, with the help of the discussed future colliders, SUSY should be discovered, if superpartners are responsible for the explanation of the $(g-2)_{\mu}$ anomaly.

the $100 \mathrm{TeV}$ hadron collider will discover SUSY if CMSSM is the correct model [58]. Our analysis is more general since we do not assume any specific model of SUSY breaking. 


\section{Acknowledgments}

MB would like to thank Thomas Hahn and Sven Heinemeyer for useful correspondence about the new version of FeynHiggs. This work is a part of the "Implications of the Higgs boson discovery on supersymmetric extensions of the Standard Model" project funded within the HOMING PLUS programme of the Foundation for Polish Science. This work has been also supported by National Science Centre under research grants DEC2011/01/M/ST2/02466, DEC-2012/04/A/ST2/00099, DEC-2012/05/B/ST2/02597. MB has been partially supported by the MNiSW grant IP2012 030272. MB thanks the Galileo Galilei Institute for Theoretical Physics and INFN for hospitality and partial support while this work was initiated. The authors are grateful to the Mainz Institute for Theoretical Physics (MITP) for its hospitality and its partial support during the completion of this work. ML was supported by the Foundation for Polish Science International PhD Projects Programme co-financed by the EU European Regional Development Fund. The authors acknowledge also hospitality of the CERN theory group during preparation of this work.

\section{A Bino contribution}

In this appendix we discuss the effects of a hypothetical very heavy higgsino, hierarchically heavier than gauginos and sleptons. There exists a contribution to $(g-2)_{\mu}$ that does not decouple in that limit. It is given by the Feynman diagram with the loop involving bino and smuon with a chirality flip occurring on the smuon line and it is approximately given by (2.3). This diagram is obviously suppressed by the smuon masses but it is proportional to the smuon mixing which, in turn, is proportional to $A_{\mu}-\mu \tan \beta$. This means that, contrary to other contributions, it grows with the higgsino mass rather than decouples. It is most effective when bino and smuon masses are close to each other (for $M_{1} \ll m_{\tilde{\mu}}$ it is suppressed by $M_{1}$ in the numerator of (2.3) while for $M_{1} \gg m_{\tilde{\mu}}$ it is suppressed by the loop function $f_{\chi^{0}}$ defined in $\left.(2.4)\right)$. In principle the $(g-2)_{\mu}$ anomaly can be explained for any value of $\tan \beta$ and the smuon and bino masses by taking appropriately large $\mu .{ }^{9}$ This is demonstrated in figure 5 . It can be seen that agreement with the $(g-2)_{\mu}$ measurement at $1 \sigma$ is possible for heavier sleptons than discussed in the previous section but at the cost of highly unnatural values of $\mu$. For example for $\tan \beta=10$ and smuon masses of $500 \mathrm{GeV}$ $(g-2)_{\mu}$ can be within $1 \sigma$ from the experimental value for $\mu \approx 20 \mathrm{TeV}$ (for light charginos satisfying the LEP limits such smuon masses would not allow for $(g-2)_{\mu}$ within $\left.1 \sigma\right)$.

A large bino contribution due to such a hierarchical spectrum is strongly disfavored by the naturalness arguments. However, it turns out that this possibility can be constrained also in a more objective way. A detailed study of that case was performed in ref. [19]. Too large values of $\mu \tan \beta$ lead to instability of the EW vacuum due to large trilinear coupling of sleptons to the Higgs. It was shown in ref. [19] that for universal slepton masses the vacuum stability implies that $(g-2)_{\mu}$ consistent with the measurement at $1 \sigma$ can be obtained only for the lightest smuon mass below about $300 \mathrm{GeV}$ (we reproduce this result,

\footnotetext{
${ }^{9}$ For simplicity of the discussion we assume $A_{\mu}=0$ but in general bino contribution is scaled by $\mu \tan \beta-A_{\mu}$ so large negative $A_{\mu}$ can enhance $(g-2)_{\mu}$ in a similar way as $\mu \tan \beta$ does.
} 


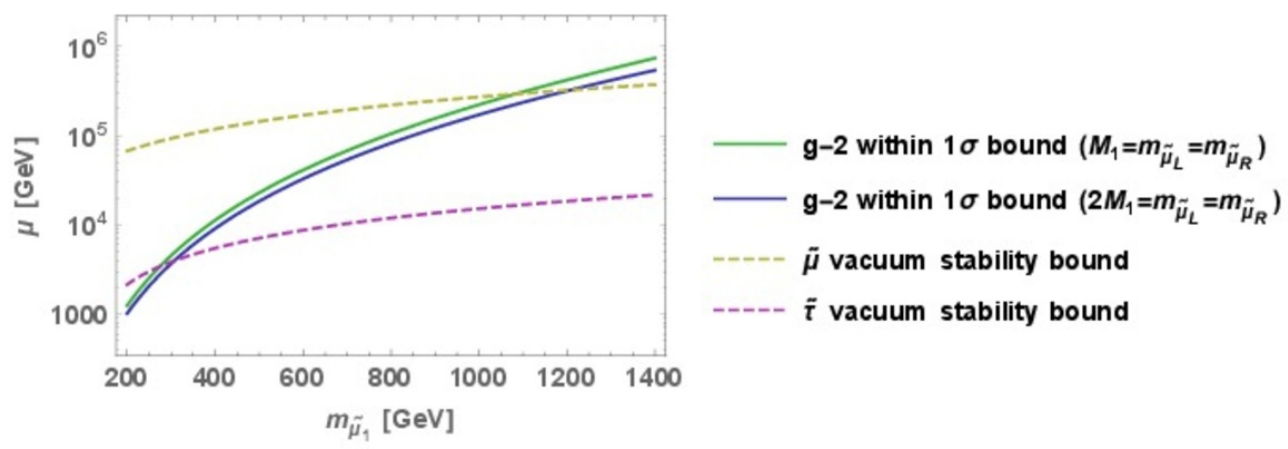

Figure 5. Minimal value of $\mu$ for $\tan \beta=10$ required for the bino contribution to be consistent with the $(g-2)_{\mu}$ measurement at $1 \sigma$ level as a function of the lightest smuon mass (solid lines). Upper bounds on $\mu$ from the EW vacuum stability in the smuon and stau directions, calculated using the formula (14) of ref. [19], are also shown (dashed lines).

using the formula (14) of ref. [19], in figure 5). This upper bound is independent of $\tan \beta$ because what matters is $\mu \tan \beta$ (of course the saturation of this bound requires heavier higgsinos for smaller $\tan \beta$ ). Moreover, it was shown in ref. [19] that most of that region of the parameter space is already excluded by the LHC searches. Only a small window of the lightest smuon masses between about 290 and $300 \mathrm{GeV}$ for a very restricted range of bino masses remains allowed. This window can be extended to about $400 \mathrm{GeV}$ assuming that the $(g-2)_{\mu}$ is brought in agreement with the measurement only at $2 \sigma$. In any case, this window will be probed at the LHC with $\sqrt{s}=13 \mathrm{TeV}$.

The vacuum stability constraint can be relaxed if the stau masses are larger than the smuon masses because then larger values of $\mu \tan \beta$ (which control the size of the offdiagonal entry of the stau mass matrix that tends to destabilize the vacuum) are allowed. In consequence, for a given value of $a_{\mu}^{\text {SUSY }}$ smuons can be heavier. ${ }^{10}$ However, if the stau masses are larger than the smuon masses by a factor bigger than about 15 (which roughly corresponds to the ratio of the tau to muon masses) the vacuum stability constraint in the muon direction becomes more stringent than that in the stau direction. In such a case $(g-2)_{\mu}$ can be within the $1 \sigma$ experimental bound for the lightest smuon mass up to about $1.2 \mathrm{TeV}$ (for so heavy smuons $\mu$ would have to be above $300 \mathrm{TeV}$ for $\tan \beta=10$ ). ${ }^{11}$ For a heavier smuon the electroweak vacuum is unstable in the smuon direction. Neither the LHC nor future lepton colliders, such as ILC or TLEP, will be able to probe $1.2 \mathrm{TeV}$ smuons. However, they could be within the reach of CLIC which aims to operate at the nominal center-of-mass energy of $3 \mathrm{TeV}$ [64]. It is also possible that such smuon masses could be probed at the future $100 \mathrm{TeV}$ collider.

It was also noticed in ref. [19] that a large non-universality between smuon and stau masses leads to a strong tension with $\mu \rightarrow e \gamma$ unless lepton flavor violation is extremely

\footnotetext{
${ }^{10}$ If the stau masses are lighter than the smuon and electron masses the bino contribution to $(g-2)_{\mu}$ is more constrained by the vacuum stability in the stau direction and the smuons have to be lighter than in the universal slepton case.

${ }^{11}$ Ref. [19] found slightly larger upper bound on the lightest smuon mass of about $1.4 \mathrm{TeV}$. The difference stems mainly from the fact that we use the value of $(g-2)_{\mu}$ based on the SM prediction of ref. [12] while ref. [19] used the result of ref. [13].
} 
small (the mass-insertion parameters should be below $\left.10^{-6}\right) .{ }^{12}$ Therefore, the bino contribution can be efficiently probed also by looking for rare decays. Similarly, the CP phase of the $\mu$ parameter has to be strongly suppressed in order to avoid constraints from the electric dipole moments.

Open Access. This article is distributed under the terms of the Creative Commons Attribution License (CC-BY 4.0), which permits any use, distribution and reproduction in any medium, provided the original author(s) and source are credited.

\section{References}

[1] M. Papucci, J.T. Ruderman and A. Weiler, Natural SUSY endures, JHEP 09 (2012) 035 [arXiv:1110.6926] [INSPIRE].

[2] N. Craig, M. McCullough and J. Thaler, Flavor mediation delivers natural SUSY, JHEP 06 (2012) 046 [arXiv: 1203.1622] [INSPIRE].

[3] M. Badziak, E. Dudas, M. Olechowski and S. Pokorski, Inverted sfermion mass hierarchy and the Higgs boson mass in the MSSM, JHEP 07 (2012) 155 [arXiv:1205.1675] [INSPIRE].

[4] G.G. Ross and K. Schmidt-Hoberg, The fine-tuning of the generalised NMSSM, Nucl. Phys. B 862 (2012) 710 [arXiv:1108.1284] [INSPIRE].

[5] L.J. Hall, D. Pinner and J.T. Ruderman, A natural SUSY Higgs near 126 GeV, JHEP 04 (2012) 131 [arXiv:1112.2703] [INSPIRE].

[6] T. Gherghetta, B. von Harling, A.D. Medina and M.A. Schmidt, The scale-invariant NMSSM and the $126 \mathrm{GeV}$ Higgs boson, JHEP 02 (2013) 032 [arXiv:1212.5243] [INSPIRE].

[7] N. Arkani-Hamed and S. Dimopoulos, Supersymmetric unification without low energy supersymmetry and signatures for fine-tuning at the LHC, JHEP 06 (2005) 073 [hep-th/0405159] [INSPIRE].

[8] G.F. Giudice and A. Romanino, Split supersymmetry, Nucl. Phys. B 699 (2004) 65 [Erratum ibid. B 706 (2005) 65] [hep-ph/0406088] [INSPIRE].

[9] G. Elor, L.J. Hall, D. Pinner and J.T. Ruderman, Yukawa unification and the superpartner mass scale, JHEP 10 (2012) 111 [arXiv:1206.5301] [INSPIRE].

[10] L.J. Hall, J.T. Ruderman and T. Volansky, A cosmological upper bound on superpartner masses, JHEP 02 (2015) 094 [arXiv:1302.2620] [INSPIRE].

[11] Muon G-2 collaboration, G.W. Bennett et al., Final report of the muon E821 anomalous magnetic moment measurement at BNL, Phys. Rev. D 73 (2006) 072003 [hep-ex/0602035] [INSPIRE].

[12] M. Davier, A. Hoecker, B. Malaescu and Z. Zhang, Reevaluation of the hadronic contributions to the muon $g-2$ and to $\alpha_{M Z}$, Eur. Phys. J. C 71 (2011) 1515 [Erratum ibid. C 72 (2012) 1874] [arXiv:1010.4180] [inSPIRE].

[13] K. Hagiwara, R. Liao, A.D. Martin, D. Nomura and T. Teubner, $(g-2)_{\mu}$ and $\alpha\left(M_{Z}^{2}\right)$ re-evaluated using new precise data, J. Phys. G 38 (2011) 085003 [arXiv:1105.3149] [INSPIRE].

[14] M. Benayoun, P. David, L. DelBuono and F. Jegerlehner, An update of the HLS estimate of the muon g-2, Eur. Phys. J. C 73 (2013) 2453 [arXiv:1210.7184] [InSPIRE].

\footnotetext{
${ }^{12}$ Correlation between $(g-2)_{\mu}$ and $\mu \rightarrow e \gamma$ was recently investigated also in [65].
} 
[15] D. Stöckinger, The muon magnetic moment and supersymmetry, J. Phys. G 34 (2007) R45 [hep-ph/0609168] [INSPIRE].

[16] S.P. Martin and J.D. Wells, Muon anomalous magnetic dipole moment in supersymmetric theories, Phys. Rev. D 64 (2001) 035003 [hep-ph/0103067] [InSPIRE].

[17] M.E. Cabrera, J.A. Casas and A. Delgado, Upper bounds on superpartner masses from upper bounds on the Higgs boson mass, Phys. Rev. Lett. 108 (2012) 021802 [arXiv:1108.3867] [INSPIRE].

[18] G.F. Giudice and A. Strumia, Probing high-scale and split supersymmetry with Higgs mass measurements, Nucl. Phys. B 858 (2012) 63 [arXiv:1108.6077] [INSPIRE].

[19] M. Endo, K. Hamaguchi, T. Kitahara and T. Yoshinaga, Probing bino contribution to muon $g-2$, JHEP 11 (2013) 013 [arXiv:1309.3065] [INSPIRE].

[20] A. Arbey, M. Battaglia, A. Djouadi, F. Mahmoudi and J. Quevillon, Implications of a 125 GeV Higgs for supersymmetric models, Phys. Lett. B 708 (2012) 162 [arXiv:1112.3028] [INSPIRE].

[21] S. Mohanty, S. Rao and D.P. Roy, Reconciling the muon $g-2$ and dark matter relic density with the LHC results in nonuniversal gaugino mass models, JHEP 09 (2013) 027 [arXiv:1303.5830] [INSPIRE].

[22] S. Akula and P. Nath, Gluino-driven radiative breaking, Higgs boson mass, muon $g-2$ and the Higgs diphoton decay in supergravity unification, Phys. Rev. D 87 (2013) 115022 [arXiv: 1304.5526] [INSPIRE].

[23] J. Chakrabortty, S. Mohanty and S. Rao, Non-universal gaugino mass GUT models in the light of dark matter and LHC constraints, JHEP 02 (2014) 074 [arXiv:1310.3620] [INSPIRE].

[24] I. Gogoladze, F. Nasir, Q. Shafi and C.S. Un, Nonuniversal gaugino masses and muon g-2, Phys. Rev. D 90 (2014) 035008 [arXiv:1403.2337] [InSPIRE].

[25] S.P. Martin, Non-universal gaugino masses from non-singlet F-terms in non-minimal unified models, Phys. Rev. D 79 (2009) 095019 [arXiv: 0903.3568] [InSPIRE].

[26] M. Badziak, M. Olechowski and S. Pokorski, Light staus and enhanced Higgs diphoton rate with non-universal gaugino masses and $\mathrm{SO}(10)$ Yukawa unification, JHEP 10 (2013) 088 [arXiv: 1307.7999] [INSPIRE].

[27] T. Moroi, The muon anomalous magnetic dipole moment in the minimal supersymmetric standard model, Phys. Rev. D 53 (1996) 6565 [Erratum ibid. D 56 (1997) 4424] [hep-ph/9512396] [INSPIRE].

[28] Particle Data Group collaboration, J. Beringer et al., Review of particle physics, Phys. Rev. D 86 (2012) 010001 [INSPIRE].

[29] ALEPH collaboration, A. Heister et al., Search for scalar leptons in $e^{+} e^{-}$collisions at center-of-mass energies up to 209 GeV, Phys. Lett. B 526 (2002) 206 [hep-ex/0112011] [INSPIRE].

[30] DELPHI collaboration, J. Abdallah et al., Searches for supersymmetric particles in $e^{+} e^{-}$ collisions up to $208 \mathrm{GeV}$ and interpretation of the results within the MSSM, Eur. Phys. J. C 31 (2003) 421 [hep-ex/0311019] [INSPIRE].

[31] L3 collaboration, P. Achard et al., Search for scalar leptons and scalar quarks at LEP, Phys. Lett. B 580 (2004) 37 [hep-ex/0310007] [INSPIRE]. 
[32] OPAL collaboration, G. Abbiendi et al., Search for anomalous production of dilepton events with missing transverse momentum in $e^{+} e^{-}$collisions at $\sqrt{s}=183$ Gev to 209-GeV, Eur. Phys. J. C 32 (2004) 453 [hep-ex/0309014] [INSPIRE].

[33] EP2 SUSY working group, http://lepsusy.web.cern.ch/lepsusy/www/sleptons_summer04 /slep_final.html.

[34] ATLAS collaboration, Search for direct production of charginos and neutralinos in events with three leptons and missing transverse momentum in $\sqrt{s}=8 \mathrm{TeV}$ pp collisions with the ATLAS detector, JHEP 04 (2014) 169 [arXiv:1402.7029] [INSPIRE].

[35] ATLAS collaboration, Search for direct production of charginos, neutralinos and sleptons in final states with two leptons and missing transverse momentum in pp collisions at $\sqrt{s}=8 \mathrm{TeV}$ with the ATLAS detector, JHEP 05 (2014) 071 [arXiv:1403.5294] [INSPIRE].

[36] CMS collaboration, Searches for electroweak production of charginos, neutralinos and sleptons decaying to leptons and $W, Z$ and Higgs bosons in pp collisions at $8 \mathrm{TeV}$, Eur. Phys. J. C 74 (2014) 3036 [arXiv:1405.7570] [INSPIRE].

[37] P. Schwaller and J. Zurita, Compressed electroweakino spectra at the LHC, JHEP 03 (2014) 060 [arXiv: 1312.7350] [INSPIRE].

[38] Fermilab E989 collaboration, G. Venanzoni, The new $g-2$ experiment at Fermilab, Frascati Phys. Ser. 56 (2012) 195 [inSPIRE].

[39] J-PARC NEW G-2/EDM EXPERIMENT collaboration, H. Iinuma, New approach to the muon g-2 and EDM experiment at J-PARC, J. Phys. Conf. Ser. 295 (2011) 012032 [inSPIRE].

[40] H.G. Fargnoli et al., Non-decoupling two-loop corrections to $(g-2)_{\mu}$ from fermion/sfermion loops in the MSSM, Phys. Lett. B 726 (2013) 717 [arXiv:1309.0980] [INSPIRE].

[41] D. Chowdhury, R.M. Godbole, K.A. Mohan and S.K. Vempati, Charge and color breaking constraints in MSSM after the Higgs discovery at LHC, JHEP 02 (2014) 110 [arXiv:1310.1932] [INSPIRE].

[42] N. Blinov and D.E. Morrissey, Vacuum stability and the MSSM Higgs mass, JHEP 03 (2014) 106 [arXiv: 1310.4174] [INSPIRE].

[43] J.E. Camargo-Molina, B. Garbrecht, B. O'Leary, W. Porod and F. Staub, Constraining the Natural MSSM through tunneling to color-breaking vacua at zero and non-zero temperature, Phys. Lett. B 737 (2014) 156 [arXiv:1405.7376] [INSPIRE].

[44] S. Heinemeyer, W. Hollik and G. Weiglein, FeynHiggs: a program for the calculation of the masses of the neutral CP even Higgs bosons in the MSSM, Comput. Phys. Commun. 124 (2000) 76 [hep-ph/9812320] [INSPIRE].

[45] T. Hahn, S. Heinemeyer, W. Hollik, H. Rzehak and G. Weiglein, High-precision predictions for the light CP-even Higgs boson mass of the minimal supersymmetric standard model, Phys. Rev. Lett. 112 (2014) 141801 [arXiv:1312.4937] [INSPIRE].

[46] ATLAS collaboration, Measurement of the Higgs boson mass from the $H \rightarrow \gamma \gamma$ and $H \rightarrow Z Z^{*} \rightarrow 4 \ell$ channels with the ATLAS detector using $25 \mathrm{fb}^{-1}$ of pp collision data, Phys. Rev. D 90 (2014) 052004 [arXiv: 1406.3827] [INSPIRE].

[47] CMS collaboration, Precise determination of the mass of the Higgs boson and studies of the compatibility of its couplings with the standard model, CMS-PAS-HIG-14-009 (2014).

[48] ATLAS collaboration, Search for neutral Higgs bosons of the minimal supersymmetric standard model in pp collisions at $\sqrt{s}=8 \mathrm{TeV}$ with the ATLAS detector, ATLAS-CONF-2014-049 (2014) [ATLAS-COM-CONF-2014-062]. 
[49] CMS collaboration, Search for neutral MSSM Higgs bosons decaying to a pair of $\tau$ leptons in pp collisions, JHEP 10 (2014) 160 [arXiv:1408.3316] [INSPIRE].

[50] ATLAS collaboration, Constraints on New Phenomena via Higgs Coupling Measurements with the ATLAS Detector, ATLAS-CONF-2014-010 (2014) [ATLAS-COM-CONF-2014-011].

[51] ATLAS, CDF, CMS, D0 collaboration, First combination of Tevatron and LHC measurements of the top-quark mass, arXiv:1403.4427 [INSPIRE].

[52] P. Draper, G. Lee and C.E.M. Wagner, Precise estimates of the Higgs mass in heavy supersymmetry, Phys. Rev. D 89 (2014) 055023 [arXiv: 1312.5743] [INSPIRE].

[53] E. Bagnaschi, G.F. Giudice, P. Slavich and A. Strumia, Higgs mass and unnatural supersymmetry, JHEP 09 (2014) 092 [arXiv: 1407.4081] [INSPIRE].

[54] M. Endo, K. Hamaguchi, S. Iwamoto and T. Yoshinaga, Muon g-2 vs. LHC in supersymmetric models, JHEP 01 (2014) 123 [arXiv:1303.4256] [INSPIRE].

[55] ILC collaboration, G. Aarons et al., International Linear Collider reference design report volume 2: physics at the ILC, arXiv:0709.1893 [INSPIRE].

[56] TLEP Design Study Working Group collaboration, M. Bicer et al., First look at the physics case of TLEP, JHEP 01 (2014) 164 [arXiv:1308.6176] [INSPIRE].

[57] T. Cohen et al., SUSY simplified models at 14, 33 and 100 TeV proton colliders, JHEP 04 (2014) 117 [arXiv:1311.6480] [INSPIRE].

[58] A. Fowlie and M. Raidal, Prospects for constrained supersymmetry at $\sqrt{s}=33 \mathrm{TeV}$ and $\sqrt{s}=100$ TeV proton-proton super-colliders, Eur. Phys. J. C 74 (2014) 2948 [arXiv: 1402.5419] [INSPIRE].

[59] T. Cohen, R.T. D'Agnolo, M. Hance, H.K. Lou and J.G. Wacker, Boosting stop searches with a $100 \mathrm{TeV}$ proton collider, JHEP 11 (2014) 021 [arXiv:1406.4512] [INSPIRE].

[60] U. Ellwanger, C. Hugonie and A.M. Teixeira, The next-to-minimal supersymmetric standard model, Phys. Rept. 496 (2010) 1 [arXiv:0910.1785] [INSPIRE].

[61] F. Domingo and U. Ellwanger, Constraints from the muon $g-2$ on the parameter space of the NMSSM, JHEP 07 (2008) 079 [arXiv:0806.0733] [INSPIRE].

[62] F. Brummer, S. Kraml and S. Kulkarni, Anatomy of maximal stop mixing in the MSSM, JHEP 08 (2012) 089 [arXiv: 1204.5977] [INSPIRE].

[63] M. Badziak, M. Olechowski and S. Pokorski, New regions in the NMSSM with a $125 \mathrm{GeV}$ Higgs, JHEP 06 (2013) 043 [arXiv: 1304.5437] [InSPIRE].

[64] CLIC Physics Working Group collaboration, E. Accomando et al., Physics at the CLIC multi-TeV linear collider, hep-ph/0412251 [INSPIRE].

[65] J. Kersten, J.-h. Park, D. Stöckinger and L. Velasco-Sevilla, Understanding the correlation between $(g-2)_{\mu}$ and $\mu \rightarrow e \gamma$ in the MSSM, JHEP 08 (2014) 118 [arXiv:1405.2972] [INSPIRE]. 